\title{
Experiencias de aplicación de técnicas cualitativas en estudios de hombres que tienen sexo con hombres*
}

\section{Experience in use of qualitative techniques for the study of men having sex with other men}

\author{
Nereida Rojo Pérez' ; Yisel Torres Rojo"' ; Luisa Álvarez Vázquez"'; Nelcy

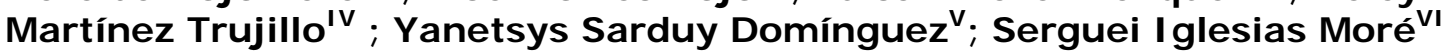 \\ 'Doctora en Ciencias de la Salud. Profesor Titular. Escuela Nacional de Salud \\ Pública. La Habana, Cuba. \\ "Máster en Educación y Promoción de Salud. Instituto de Medicina Tropical "Pedro \\ Kourí". La Habana, Cuba. \\ II'Doctora en Ciencias Económicas. Investigador Titular y de Mérito. Instituto \\ Nacional de Endocrinología. La Habana, Cuba. \\ IVMáster en Salud Pública. Escuela Nacional de Salud Pública. La Habana, Cuba.

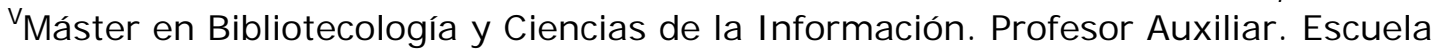 \\ Nacional de Salud Pública. La Habana, Cuba. \\ ${ }^{V^{\prime}}$ Máster en Psicología de la Salud. Profesor Auxiliar. Centro para el Desarrollo \\ Académico sobre Drogodependencias. La Habana, Cuba.
}

\section{RESUMEN}

La investigación cualitativa es una herramienta metodológica útil para el estudio de los hombres que tienen sexo con otros hombres como grupo más vulnerable a la infección por VIH en Cuba, por lo que es necesario conocer sus principios, metodologías y los resultados de su aplicación. Los objetivos propuestos fueron caracterizar las experiencias cognoscitivas, éticas y metodológicas derivadas de los estudios cualitativos realizados para el abordaje del tema VIH-sida en hombres cubanos que tienen sexo con otros hombres. Se empleó un proceso de sistematización de experiencias, a partir de tres investigaciones desarrolladas entre los años 2003-2008, donde el objeto de estudio fueron los hombres que tienen sexo con otros hombres y se usaron técnicas cualitativas. Se describe el contexto y el plan de investigación seguido en las experiencias analizadas. Se hace un análisis crítico de las principales técnicas utilizadas en el abordaje de los hombres que tienen sexo con otros hombres, de su valor metodológico, sus implicaciones éticas y de los resultados obtenidos en términos de conocimientos acerca del grupo. Asimismo, se presentan las lecciones aprendidas en el proceso. Los resultados obtenidos indican la importancia del uso de la metodología cualitativa, para el desarrollo de investigaciones, programas y políticas dirigidos a la prevención y el control de las infecciones de transmisión sexual y el VIH-sida en el país, en grupos vulnerables de escasa visibilidad y alcance como los hombres que tienen sexo con otros hombres.

Palabras clave: Hombres que tienen sexo con otros hombres, $\mathrm{VIH}$-sida, investigación cualitativa, sistematización de experiencias. 


\begin{abstract}
The qualitative research is a useful methodological tool for the study of men having sex with other men as the most vulnerable group to HIV infection in Cuba; therefore, it is necessary to know about its principles, methods and the results of its application. The objectives of this paper were to characterize the cognitive, ethical and methodological experience from the qualitative studies carried out to approach HIV-AIDS in Cuban men having sex with other men. A process of experience systematization was included on the basis of research works conducted from 2003 to 2008, where the object of study was men having sex with other men, and also qualitative techniques were used. Critical analysis was made on the main techniques present in the approach to men having sex with other men, their methodological value and ethical implications as well as the resulting knowledge about this group. Likewise, the lessons learned throughout the process were presented. The outcome revealed the importance of the qualitative methodology for the development of research, programs and policies aimed at STI and HIV-AIDS prevention and control in low visibility and scope groups as that of men having sex with other men.
\end{abstract}

Key words: Men having sex with other men, HIV-AIDS, qualitative research, experience systematization.

\title{
I NTRODUCCI ÓN
}

Hombres que tienen sexo con otros hombres (HSH) es un término que se utiliza para tipificar las distintas expresiones de la conducta de los varones que tienen alguna preferencia por las relaciones sexuales con personas de su mismo sexo, llámense homosexuales, bisexuales, travestidos, transexuales, transgénero o cualquier otra de esta naturaleza, de carácter temporal o permanente. ${ }^{1}$ Las relaciones sexuales entre hombres, aunque han existido en prácticamente todas las sociedades conocidas, antiguas y modernas, han sido por lo general estigmatizadas; al cierre del 2007, Amnistía Internacional reconocía 70 países donde estos comportamientos son aún penalizados por la ley. ${ }^{2}$

Los HSH constituyen una de las minorías sociales que más atención han generado a lo largo del desarrollo de la humanidad como fuente de conflictos y de interés humano, científico y social, en su lucha por adquirir iguales derechos y estatus que los heterosexuales. Proceso que se visualiza con más nitidez desde mediados del siglo XX, fundamentalmente en Norteamérica y Europa, donde sus líderes y organizaciones, imbuidos del influjo de los grandes procesos de liberación nacional en el mundo y de la lucha por alcanzar sus derechos que protagonizaban otras minorías, ${ }^{3}$ inician un proceso de empoderamiento y de militancia que unido a los resultados de las investigaciones biomédicas y sociales, condujeron a que la Asociación de Psiquiatría de Estados Unidos eliminara en 1973 a la homosexualidad de la lista de desórdenes mentales. ${ }^{4}$ 
Este proceso de activismo gay tuvo manifestaciones y repercusiones en el orden sociolegal como el reconocimiento del derecho a la libre unión y el acceso al matrimonio en determinados estados y países, ${ }^{5,6}$ con lo cual se esperaba una verdadera revolución en este campo.

Sin embargo, la aparición de la epidemia de VIH-sida en la década de los 80 del pasado siglo, identificada en sus inicios como una enfermedad de homosexuales, volvió a colocar a esta minoría en una situación de vulnerabilidad medicosocial y aún cuando esa tesis ha sido descalificada por la evidencia científica, persisten rasgos de homofobia en las sociedades contemporáneas y una doble estigmatización, por su conducta sexual y su potencial condición de seropositivo.

En Cuba, la homosexualidad ha sido también históricamente rechazada dados los patrones heterosexistas de la sociedad, mezcla cultural del machismo español y africano. ${ }^{7}$ El proceso de apertura se inicia más lentamente, primero en las artes, la literatura, el cine, las ciencias sociales y de la salud, lo que ha permitido una mayor visibilidad social de esta minoría y un amplio debate al respecto.

La epidemia de VIH cubana se clasifica como concentrada, por tener muy baja prevalencia en la población general y las mayores tasas de infección en el subgrupo de hombres que tienen sexo con hombres, con un patrón dominante de transmisión sexual en el cual el porcentaje de varones era de $80,6 \%$ y de $19,4 \%$ en mujeres, al cierre del $2007 .^{8}$

En el campo de la prevención del VIH las intervenciones han ido evolucionando de la población general hacia el principal grupo afectado, los HSH, para elevar sus conocimientos acerca de la protección sexual, sensibilizar a la población y así disminuir el estigma y la discriminación. ${ }^{9}$ No obstante, se carece de datos precisos, acerca de este grupo poblacional en relación con los factores que determinan sus comportamientos de salud, dada la naturaleza esencialmente privada de la vida personal y la carencia de investigaciones pertinentes acerca del punto de vista de los varones en el campo de la salud sexual y reproductiva. ${ }^{10}$

Las características descritas de la epidemia cubana demuestran la necesidad de entender los determinantes del comportamiento sexual de esta minoría, para crear estrategias educativas, políticas y programas efectivos que logren a mediano plazo disminuir su situación de vulnerabilidad, sin afectar al resto de las capas del entramado social. Para ello es fundamental, el uso de la investigación cualitativa.

La investigación cualitativa, es una de las formas que asume la investigación social para estudiar el comportamiento humano, las relaciones sociales y el contexto sociocultural del hombre. Es una visión paradigmática que centra su atención en los valores, las concepciones y la interpretación que de la vida diaria, del día a día, tienen los que la viven. ${ }^{11}$

Es un proceso inductivo de indagación que implica recolectar y analizar datos de la vida cotidiana y del medio local donde "el micro mundo del hombre" adquiere una nueva dimensión, al interpretar lo que allí acontece sobre la base de nuevos datos o nuevos posicionamientos teóricos. Como en toda investigación las posibilidades de su uso efectivo, dependerá de la naturaleza del problema, del marco epistémico del investigador, del rigor metodológico observado y de su pertinencia social. ${ }^{12}$

El propósito del presente estudio es caracterizar las experiencias cognoscitivas, éticas y metodológicas, derivadas de los estudios cualitativos realizados para el abordaje del tema VIH-sida en HSH cubanos. 


\section{MÉTODOS}

Se usó como metodología de trabajo el proceso de sistematización de experiencias ${ }^{13}$ (Bustillo G. Sistematización. Selección de lecturas. La Habana: Asociación de Pedagogos de Cuba), que consiste en la interpretación crítica de los resultados obtenidos en la aplicación de técnicas cualitativas, sobre la base de ordenar y reconstruir el acto de indagación desde su concepción, con lo cual se persigue explicar la estructura y lógica del proceso, los métodos empleados, los factores que intervinieron y sus interrelaciones.

Para ello, se hizo una revisión documental de estudios cubanos donde el objeto central fuesen los HSH y se hubiesen usado técnicas cualitativas como método básico o complementario. Se consideraron las bases de datos de la biblioteca de la Escuela Nacional de Salud Pública (ENSAP) que contiene las referencias bibliográficas de las tesis de grado de los egresados de seis maestrías que imparte la institución: salud pública, atención primaria de salud, psicología de la salud, educación médica superior, promoción y educación para la salud y economía de la salud; de las especialidades de bioestadística e higiene y epidemiología, así como del doctorado en ciencias de la salud.

Otras fuentes de información fueron el registro de investigaciones cerradas de la ENSAP, así como del Programa Ramal de Investigaciones en Sistemas y Servicios de Salud (ISSS) que la Escuela coordina y el portal Scielo-Cuba que contiene la producción de las revistas médicas cubanas. En todos los casos la búsqueda se hizo a través de la técnica de palabras clave y para ello no se delimitó universo temporal. Finalmente se incluyó el período 2003-2008.

El análisis constó de dos etapas: una primera de trabajo de mesa donde una vez identificados los documentos que cumplían los requisitos de entrada, se hizo una lectura profunda y pormenorizada de los textos escritos, con el objetivo de obtener la información primaria sobre los órdenes cognoscitivos, metodológicos y éticos relacionados con el grupo de estudio, los instrumentos, su aplicación, el desempeño de la función de investigador en el trabajo de campo y el tratamiento de los datos.

La segunda etapa consistió en una discusión grupal, donde mediante un proceso eminentemente participativo, los autores ordenaron las categorías, revisaron críticamente los hallazgos, así como sus propias percepciones. La valoración integrada de los resultados fue el eslabón fundamental, para el desarrollo de las lecciones aprendidas así como de las consideraciones finales.

Se identificaron cuatro investigaciones cuyo objeto estaba centrado en los HSH y en todas se emplearon técnicas cualitativas, de ellas una tesis de la maestría de psicología de la salud no fue considerada porque no cumplía los criterios de inclusión y databa de más de 10 años.

\section{RESULTADOS}

El objeto de estudio quedó conformado por tres experiencias, dos pertenecían a un proyecto de investigación en fase de cierre (Rojo N, Pérez J, Llanusa S, Álvarez L, Sanabria G, Louro I, et. al. Contribuciones para una estrategia multisectorial integrada para la prevención y control del problema de salud pública VIH-sida en Cuba. 2005-2008. Proyecto Escuela Nacional de Salud Pública del Programa Ramal de Enfermedades Transmisibles), y la otra era una tesis de la maestría de promoción y educación para la salud. ${ }^{14}$ Todas provenían de la Escuela Nacional de Salud Pública y en las cuales habían participado los autores del presente trabajo. En 
los tres estudios, los objetivos estaban dirigidos a entender las actitudes, motivaciones, comportamientos, percepciones de sí mismos y de riesgo frente al $\mathrm{VIH}$ de los HSH, lo que los situaba en una perspectiva interpretativa etnográfica o comprehensiva.

\section{Aspectos generales de las experiencias}

Contexto de las investigaciones. Se efectuaron en cinco de las 14 provincias del país, que representaron las tres regiones en que tradicionalmente se ha dividido: Occidente, con Ciudad de La Habana, Provincia Habana y Matanzas. El Centro con Villa Clara y el Oriente por Holguín. Los estudios aunque comprendían localidades urbanas y rurales, el volumen mayor de sitios y HSH se localizó en las capitales municipales o provinciales, ámbitos con un mayor nivel de tolerancia social. El período comprendido, es una etapa en que se ejecutaron en el país un conjunto de proyectos de prevención del VIH desde los diferentes sectores sociales dirigidos a los grupos vulnerables incluido el proyecto "HSH", como parte de la sociedad civil y en apoyo a las acciones del Programa Nacional de Prevención y Control del VIH, por lo cual el tema ha sido tratado en los medios masivos de difusión a través de la ficción y/o del testimonio, cine-debates, peñas, grupos especializados y alcanza su clímax con la celebración de la semana de la diversidad sexual.

Equipos de investigación. Estuvieron conformados por profesionales de distintas disciplinas, instituciones y provincias; con conocimientos, experiencia de trabajo y habilidades en el uso de la metodología cualitativa. La mayoría eran mujeres y promotores pares de distintos grupos de edades.

Plan de la investigación. Las técnicas utilizadas para la búsqueda de información fueron: grupos de discusión con promotores pares, entrevistas a profundidad en $\mathrm{HSH}$, entrevistas de salida y observación no participante en sitios de encuentro.

Los instrumentos fueron cuidadosamente elaborados por el equipo de investigación y validados por expertos reconocidos en la temática HSH y VIH. Para su aplicación los investigadores se sometieron a un proceso de capacitación que incluía el repaso de los requisitos de cada técnica específica y el entrenamiento per se con dramatizaciones en que se controlaba el tono de la voz, la gestualidad y el vocabulario, para evitar cualquier forma de expresión que pudiese ser considerada por los sujetos como un juicio de valor acerca de sus conductas y dañara la comunicación, con lo cual se daría al traste con la intención de hallar respuestas lo más fidedignas posibles a las preguntas realizadas.

La puesta a punto de los instrumentos, de la capacidad de los investigadores y de la estrategia de trabajo de campo, se hizo a partir de pruebas pilotos realizadas en lugares reales, con características similares al entorno de la investigación.

La forma básica de procesamiento de los datos fue la codificación a partir de categorías preestablecidas y otras surgidas en el proceso de análisis. Como métodos para garantizar la fiabilidad de la información se usó la biangulación y/o la triangulación metodológica, cruce de información proveniente de dos o tres técnicas y fuentes, respectivamente.

Ética. Se observó particular cuidado por los autores de cada estudio, en los aspectos relacionados con la obtención del consentimiento de forma oral o escrita, la selección, libre expresión y protección de la fuente de información, la 
preservación, custodia y uso de los datos, así como con los requisitos y características de los entrevistados, en los cuales se valoraba que fuesen personas sin rasgos evidentes de enfermedad o discapacidad mental.

\section{Valoración de las principales técnicas aplicadas}

Grupos de discusión con promotores pares. Técnica que consiste en usar la interacción del grupo para obtener respuestas a fondo acerca de sus representaciones sociales, comprender sus relaciones y evaluar el significado que determinadas acciones tienen sobre las personas a las cuales van destinadas, como son las políticas públicas. ${ }^{12}$ Los participantes de los grupos de discusión previamente habían recibido la solicitud de consentimiento, que aceptaron. Cada sesión estaba dirigida por un moderador, un relator y un observador, el último velaba por el tiempo. Estas sesiones eran precedidas de una explicación del estudio y se hacia una breve presentación de los integrantes de los grupos de discusión. En la presentación cada participante, daba sus datos generales y por convención, se identificaban con el nombre del municipio de donde procedían. En general, la actividad se realizaba en locales de instituciones de salud y los participantes, se colocaban en forma circular incluido el moderador, para crear un ambiente adecuado, sin distancias.

Según lo establecido, los grupos de discusión eran relativamente homogéneos y se garantizaba la regla práctica de realizar por lo menos dos grupos según cada variable definitoria. Se crearon dos grupos de discusión en cada territorio estudiado y los participantes oscilaron entre 10-12, provenientes de los distintos municipios y estratos de HSH. Los temas abordados fueron:

- Condiciones de trabajo. Los promotores son voluntarios previamente entrenados, las actividades por lo general transcurren en horario nocturno, en un ambiente que no siempre es propicio y donde han sido incluso objeto de agresiones. Otra dificultad en el trabajo es la movilidad del grupo y de sus sitios de reunión, lo que puede responder a presiones sociales de la comunidad aledaña o decisión de sus miembros, entre otras causas, como el llamado "pastoreo": ... a nosotros nos van empujando de un lugar a otro...y nosotros nos movemos con la corriente (GD1)**.

- Alcance de las acciones. Los promotores reconocieron que aún no podían llegar con su mensaje a todos los grupos: ... Jos bisexuales son un grupo al que no se puede llegar...Tampoco .... a los que tienen alto poder adquisitivo porque no van a esas actividades, ni a los intelectuales que tienen un prestigio que cuidar y menos a los que practican el sexo transaccional... (GD6).

- Necesidades percibidas. En el abordaje de los HSH es necesario tener en cuenta: ...trabajar con las familias de los jóvenes con una orientación sexual diferente, para logar instruirlos en prácticas seguras...Formar promotores varones para que los heterosexuales y los bisexuales se sientan identificados (GD9)...Hay que trabajar con ciencia y con conciencia, no es solo repartir condones... (GD5).

- Estigma y discriminación. En los HSH hay que dialogar sobre la aceptación en la sociedad, no se pueden tratar solo porque es un problema de salud (GD2).

- Prevención de la infección. ...Ja gente se ha acostumbrado a que si no le damos el condón, no se protegen...Hay que trabajar en la sensibilización de las personas para su auto cuidado... (GD7). 
- Satisfacción con el trabajo que realizan. Este trabajo de orientación ha servido para elevar la autoestima...también como terapia porque se ha podido participar y sentir que se ha sido importante para alguien alguna vez en la vida (GD6).

Entrevistas a profundidad. Es una técnica intensiva que se conceptualiza como un proceso heurístico que intenta obtener información detallada y analítica de las actividades y creencias de los miembros de un grupo, desde su perspectiva. ${ }^{11} \mathrm{En}$ los estudios de HSH analizados, la aplicación de esta técnica estuvo dirigida a identificar actitudes y valoraciones acerca de sí mismos y del grupo, estratos que lo conforman, la percepción de sus funciones sociales, de sus comportamientos en términos de prevención de VIH y experimentación de sentimientos de estigma y discriminación individual o social.

En el primer estudio se hicieron entrevistas a profundidad del tipo tradicional. En el segundo y el tercero, del tipo entrevista enfocada, ${ }^{15}$ es decir que aborda situaciones concretas. En los entrevistados el rango de edad osciló entre los 15 y los 66 años. En general tenían una conducta homosexual reconocida, ya fuese por su familia, sus pares o amigos, la cual verbalizaban y/o exteriorizaban públicamente. Solo se tuvieron cinco casos de los llamados de "closet" que hubo que entrevistar en casas particulares. Los resultados derivados de las categorías de análisis expresaron:

- Autovaloración de sí mismos. Los entrevistados se reconocen personas normales, cuya única diferencia es que prefieren las relaciones íntimas con personas de su mismo sexo: soy homosexual, pero me defino como una persona normal (T4)***.

- Estratificación del grupo. En el interior del grupo existe un conjunto de capas o segmentos, cuya denominación puede variar de una región a otra y de un período a otro. Por ejemplo, pueden ser: "clásicos", "buenos", "bajitos", "aguas mansas" y "de closet o cerrados". Está el gay que es correcto, el gay que es fuerte, el que se viste de mujer, el que es más o menos... (T4). Ello acota la disimilitud de proyecciones culturales, niveles educacionales, ocupacionales y sociales con tendencias y representaciones de la sexualidad y de su ejercicio, que van desde las conductas sexualmente responsables hasta comportamientos de alto riesgo como el cambio frecuente de pareja, las relaciones fortuitas con personas desconocidas, el sexo transaccional, así como las relaciones homobisexuales sin protección, entre otras.

- Iniciación y rechazo familiar. Cuando yo dije en mi casa que era homosexual... por poco me matan... (T9). Yo creo que si mi mamá se llegase a enterar de lo que soy es capaz de matarme o matarse... (T1).

- Relaciones homobisexuales. Aunque la mayoría de los entrevistados relató que al momento de la entrevista, solo tenía relaciones sexuales con hombres, alrededor de la cuarta parte mantenía relaciones con mujeres de forma estable u ocasional. ...Muchos tienen mujer y salen a tener relaciones con hombres y no se protegen.

- Percepción de rechazo. La situación historicocultural pesa en los entrevistados y muchos enfrentan con crudeza los prejuicios sobre la homosexualidad. ...Para mí venir aquí significa ser yo mismo, pues cuando llega la noche me siento libre, me siento yo (T5). A nivel social perciben cambios y menos manifestaciones de rechazo. Villa Clara y Ciudad de La Habana, se reconocen como los lugares de mayor aceptación de los HSH. ...Santa Clara es una ciudad peculiar. . hay mucha civilización, es una ciudad muy alante, incluso no por el hecho de ser un municipio deja de estar a la par de La Habana (T3). 
Observación no participante en sitios de encuentro. El observador conserva su identidad y desde fuera, a partir de la información que escucha y ve, intenta conocer y comprender la dinámica asociada a estos lugares, donde los HSH se reúnen para iniciar amistades o relaciones de pareja y que son aprovechados por los promotores, para brindar servicios de educación y prevención en ITS/VIH. En las mismas se producen distintas y aceleradas dinámicas que escapan a la percepción de un solo investigador por avezado que sea.

Un sitio de encuentro se puede ubicar en el centro de la vida de la ciudad o los municipios, pero la mayoría se encuentra en las periferias, alejados de las vías de comunicación y por tanto de difícil acceso, sin iluminación y escasa o nula actividad de otro tipo, por lo cual existe la posibilidad real de agresión.

Es notable la disimilitud de lugares que se usan, entre otros: un parque, una avenida, una terminal, un monte, un camino, un edificio derruido, una zona de playa de río. En ese sentido, es de destacar la labor de los promotores que llegan a veces por sus medios a trasmitir el mensaje de protección y ofrecer condones y las mejores formas para su uso.

En su dinámica se observa que inician su actividad avanzada la noche y aunque muchos de ellos llegan solos, es frecuente el arribo de grupos de iguales y un carro o más de un escurridizo que aparentemente no tienen nada que ver con el lugar, pero en realidad están buscando sexo.

Entrevistas de salida. Esta es una técnica de la evaluación rápida participativa, la cual permite identificar el problema y focalizar su solución, acorde con los criterios del grupo. Se les pedía a los HSH que salían de los sitios de encuentro, de peñas culturales u otra actividad promocional que estuviese efectuándose durante la semana de trabajo de campo, que se detuvieran un momento y que mencionaran si así lo deseaban, los factores de satisfacción e insatisfacción con las actividades de prevención del VIH que habían tenido lugar. Los tres elementos que más satisfacción les reportaban eran la distribución de condones, lubricantes y materiales educativos y la organización de las sesiones educativas (participación de artistas, actividades de transformistas y juegos lúdicos, entre otros). Los dos elementos que más insatisfacción le provocaban eran las dificultades con la accesibilidad al lugar y la no aceptabilidad de ellos en algunos sitios.

\section{Lecciones aprendidas}

Las actividades de intervención que se diseñen para fortalecer el aprendizaje de los $\mathrm{HSH}$ en términos de fomentar una conducta sexual responsable deben ser segmentadas teniendo en cuenta los diferentes estratos o subgrupos que se han identificado a través de las técnicas cualitativas entre los HSH cubanos, porque su percepción de salud y sus proyecciones comportamentales están indisolublemente vinculadas con ellos.

Existen subgrupos de HSH, por lo general quienes tienen una orientación homosexual reconocida, abiertos al dialogo con valoraciones críticas y autocríticas desarrolladas, los cuales se pueden abordar en el escenario habitual donde transcurre su vida, mientras que existen otros en los cuales es imprescindible la intermediación de pares o de personal de salud que se hayan hecho acreedores de su confianza. 
El acercamiento típico de la investigación antropológica al grupo HSH requiere de un proceso de entrenamiento especial de los investigadores en el manejo del tema y del grupo, pues la no familiarización con sus códigos, expresiones corporales y estilos de vida pueden introducir sesgos o barreras en el proceso de indagación e interpretación de los resultados.

La entrevista en profundidad enfocada resultó una técnica muy efectiva así como la observación cruzada, varios investigadores sobre un objetivo común. Los entrevistadores mejor aceptados por el grupo son las mujeres jóvenes y sus propios pares.

En la aplicación de las técnicas se constató un mayor alcance y efectividad cuando eran ejecutadas en horario nocturno, pues los HSH son una población para la cual las noches tienen una significación especial en su vida y sus actividades.

\section{DISCUSIÓN}

La sistematización de experiencias es un proceso orientado a distintos objetivos, como puede ser un momento de la investigación, para valorar los cambios y replantear objetivos, así lo señalan Crespi y colaboradores. ${ }^{15}$ En ella confluyen diferentes perspectivas de la investigación-acción, de los proyectos de intervención y desarrollo social, así como de los métodos de la educación popular.

Dichas metodologías de investigación desde la perspectiva epistemológica se centran en la comprensión de una realidad, resultado de un proceso de evolución histórica y vista a partir de la lógica y el sentir de sus protagonistas, en el caso que se trata, los HSH en el marco de la prevención de la infección por VIH.

A tenor con los resultados se debe destacar la escasez de trabajos publicados o investigaciones relacionadas con este grupo poblacional, aun cuando el uso de fuentes secundarias para la captura del dato base implica reconocer que pueden existir algunos no registrados o detectados, por el momento en que se levantó la información, inadecuada clasificación u otros. Esta situación no se corresponde con la elevada vulnerabilidad de este grupo frente a la infección por VIH y la prioridad de hallar respuesta a las interrogantes al respecto.

La investigación cualitativa, está llamada a profundizar en los determinantes psicológicos y sociales que limitan la efectividad de las intervenciones, de ahí la importancia de compartir las experiencias al respecto.

El proceso de sistematización permitió corroborar la pertinencia de las técnicas utilizadas, ya que se obtuvo un volumen considerable de información que denota en sus miembros una gran necesidad y disposición de expresar sus criterios, creencias, sus valoraciones y su sentir. Se observó que pueden ser personas muy críticas con el entorno y consigo mismos; la mayoría de los HSH, se sentían cómodos para establecer el diálogo y alimentan grandes esperanzas de ser reconocidos en la sociedad, a partir de sus acciones.

En la entrevista a profundidad y el grupo de discusión se usó una guía no estructurada, abierta que permitía estimular la conversación avanzando de las interrogantes más sencillas hacia las más difíciles hasta completar la información que demandaba el propósito enunciado. Los entrevistadores trabajaron por lo general en pareja, auxiliados a veces por la grabadora. En el grupo de discusión se trabajó en tríos: moderador, relator y observador. Ambos, moderador y entrevistador debían lograr un proceso de comunicación abierto, flexible, informal, 
fluido y creativo para lograr la empatía, como premisa necesaria para mantener la discusión centrada en el tema y progresar.

Cada casete o folio de papel se identificó con un código asignado a la persona, el grupo a que pertenecía, el lugar donde fue aplicada la técnica, la fecha y la hora. Los textos se transcribieron fielmente y en el menor tiempo posible, con las observaciones de quienes las habían realizado que debían quedar identificados también. A continuación se procedía a codificarlos y a través de la inducción analítica se construyeron las categorías y proposiciones teóricas que sirvieron de sustrato a las tesis o informes de investigación.

La entrevista en los sitios de encuentro y otros lugares públicos, si bien permitió mayor espontaneidad, inclusión de personas provenientes de diferentes estatus y tendencias, sin afeites ni preparación previa, tuvo como limitantes el tiempo y las condiciones ambientales y sociales, pues estos espacios se pueden ver afectados, en dependencia de su ubicación geográfica por el ruido, iluminación, temperaturas y lluvia, entre otros elementos.

Los sitios de encuentro pueden coincidir o ser la antesala de sitios de sexo proclives al mantenimiento de relaciones sexuales fortuitas, en condiciones no tradicionales, donde es difícil negociar el condón. El silencio y la oscuridad pueden ocultar algunas sinuosidades de la sociedad, como el ejercicio del sexo transaccional, lo cual coincide con los resultados de estudios similares en otros países. ${ }^{16,17}$

El uso de la metodología cualitativa y la triangulación de fuentes y técnicas permitió a los investigadores obtener una caracterización del grupo sobre sí mismos, conocer sus opiniones y valoraciones, en función de los distintos segmentos o estratos que comprende, en relación con sus iguales, del trabajo de prevención de $\mathrm{VIH}$ y de aquellos factores que los colocan en situación de riesgo personal y vulnerabilidad social.

\section{CONSI DERACI ONES FI NALES}

La revisión de las experiencias en el uso de técnicas cualitativas relacionadas con el tema HSH-VIH-sida denota que ha sido insuficientemente abordado desde el campo de la salud pública, lo cual no se corresponde con la naturaleza ni la dimensión que este problema tiene en el contexto nacional.

El desarrollo de políticas, programas y proyectos prioritarios relacionados con la salud sexual y reproductiva de la población, entre ellos el control de las ITS y el $\mathrm{VIH}$-sida, requiere del estudio científico de los HSH y su problemática a través del uso de la metodología cualitativa como técnica única o combinada, para lo cual los resultados obtenidos en términos de conocimientos acerca del grupo y experiencias metodológicas en su manejo, pueden servir de base.

\section{Agradecimientos}

A los entrevistadores que compartieron las largas jornadas nocturnas, llenos de entusiasmo. 
A los HSH que nos brindaron sus valiosos testimonios.

A quienes transcribieron y revisaron los textos.

\section{REFERENCI AS BI BLI OGRÁFICAS}

1. UNESCO. Guidelines on language and content in HIV and AIDS. Related Materials. Paris: UNESCO; 2006.

2. Weblslam. 70 países consideran la homosexualidad como un delito [sitio en Internet]. [citado 7 Mar 2009]. Disponible en: http://www.webislam.com/?idn=159

3. Guidens A. Sociología. 2da ed. Madrid: Alianza Universidad Textos; 1997.

4. Bailey J M. Commentary: homosexuality and mental illness. Arch Gen Psychiatry. 1999; 56:876-80.

5. Wikipedia. Matrimonio entre personas del mismo sexo [sitio en Internet]. [citado 9 Mar 2009]. Disponible en: http://es.wikipedia.org/wiki/Matrimonio_entre_personas_del_mismo_sexo

6. Balsam KF, Beauchaine TP, Rothblum ED. Three-year follow-up of same-sex couples who had civil unions in Vermont, same-sex couples not in civil unions, and heterosexual married couples. Developmental Psychol. 2008;44(1):102-16.

7. Torres Y, Rojo N, García R, Ochoa R, Álvarez I, Gascón B, et. al. Evaluación del proyecto de prevención de ITS-VIH para hombres que tienen sexo con otros hombres, desde la visión de beneficiario. Villa Clara 2003. En: Ochoa R, Rojo N, editores. Investigaciones sobre VIH en el contexto de la salud pública cubana. La Habana: Centro Nacional de Prevención de las ITS-VIH/SIDA;2006. p.115-31.

8. Miranda O. Desigualdades de salud en la infección por el virus de la Inmunodeficiencia Humana en Cuba durante los años 1986-2007 [tesis]. La Habana: Escuela Nacional de Salud Pública;2008.

9. Ochoa R. Bases teórico-metodológicas de la estrategia educativa en VIH/sida en Cuba.1986-2007 [tesis]. La Habana: Escuela Nacional de Salud Pública;2008.

10. Álvarez L, Calero J, León E. Salud sexual y reproductiva desde el punto de vista del varón. Rev Cubana Salud Pública. 2006;32(1):24-31.

11. Rojo N. Investigación cualitativa. Aplicaciones en salud [CD-ROM]. La Habana: Escuela Nacional de Salud Pública; 2005. [En: Tronco común de las maestrías con perfil de salud pública de la Escuela Nacional de Salud Pública].

12. Merton R. La entrevista en profundidad. En: Mercado J, editor. El proceso de análisis de los datos en una investigación sociocultural en salud. Guadalajara: Universidad de Guadalajara; 2000.

13. Ulin P, Robinson E, Tolley E. Investigación aplicada en salud pública. Métodos cualitativos. Washington: OPS; 2006. [Publicación Científica y Técnica No. 614].

14. Torres Y. Evaluación del Proyecto de Prevención de VIH-sida para hombres que tienen sexo con hombres. Villa Clara 2003 [tesis]. La Habana: Escuela Nacional de Salud Pública; 2004. 
15. Crespi L, Domínguez AP, Muscio L, Obach M, Pérez $M$, Portela $L$, et. al. La sistematización como proceso. Algunas reflexiones [monografía en Internet]. [citado 9 Mar 2009]. Disponible en:

http://www. inta.gov.ar/cipaf/inst/doc/sistematizacionpamp.pdf

16. Fernández-Dávila P. Amigos con derecho a roce: una oportunidad para contraer la infección por el virus de la inmunodeficiencia humana en hombres homo/bisexuales con prácticas sexuales de alto riesgo. Gac Sanit [serie en Internet].2007 Dic. [citado 7 Mar 2009];21(6). Disponible en:

http://www.infodoctor.org: $8080 /$ uid $=18001660$

17. Posada IC, Gómez Arias RD. Mercado y riesgo: escenarios de transmisión del VIH entre hombres que tienen sexo con otros hombres. Medellín, 1993-2006.

Colombia Médica. 2007; 38(3): 222-36.

Recibido: 6 de octubre de 2008.

Aprobado: 29 de junio de 2009.

Nereida Rojo Pérez. Escuela Nacional de Salud Pública. Calle Línea esq a I. EI Vedado 10 400. La Habana, Cuba.

E-mail: nereida@ensap.sld.cu

* Presentado en el Simposio "Arnaldo Tejeiro Fernández", Investigación Cualitativa en Salud Pública, 23 de junio de 2009.

**GD1: Grupo de discusión y número.

*** (T4): Testimonio y número. 